\title{
Advising an Appropriate Forecasting Method for a Snacks Item (Biscuit) Manufacture Company in Bangladesh
}

\author{
Nandita Barman ${ }^{1 *}$, M. Babul Hasan ${ }^{2}$ and Md. Nayan Dhali ${ }^{3}$ \\ ${ }^{1}$ Department of Information and Communication Technology, Bangladesh University of Professionals (BUP), Mirpur, \\ Dhaka-1216, Bangladesh \\ ${ }^{2}$ Department of Mathematics, Dhaka University, Dhaka-1000, Bangladesh \\ ${ }^{3}$ Department of Textile Engineering, Northen University, Dhaka-1213, Bangladesh
}

( Received: 25 August 2017 ; Accepted : 20 November 2017)

\begin{abstract}
In this paper, we study the most appropriate short-term forecasting methods for the newly launched biscuit factory produces different types of biscuits. One of them is nut-orange twisted biscuits. As it is a newly launched biscuit factory, it does not use any scientific method to find future demand of their products to produce for the purpose of sales. Having an error free production as well as a good invent ory management we try to find an appropriate forecasting method for the sets of data we analyzed for that specific production. Several forecasting methods of time series forecasting such as the Moving Averages, Linear Regression with Time, Exponential Smoothing, Holt's Method, Holt-Winter's Method etc. can be applied to estimate the demand and supply for these companies. This paper focuses on selecting an appropriate forecasting technique for the newly launched biscuit company. For this, we analyze Exponential Smoothing method as used to time series. We observe from the empirical results of the analysis that if the data has no trend as well as seasonality, Exponential Smoothing Forecasting Method processes as the most appropriate forecasting method for the factory. If the data experiences linear trend in it then Holt's Forecasting Method processes as the most appropriate forecasting method for the sets of data we analyzed.
\end{abstract}

Keywords: Exponential Smoothing; Smoothing Constants; Forecast Error.

\section{Introduction}

Forecasting is a way for envisaging future demand based on previous time series data. This process is very useful for grouped data rather than individual data. Forecasting process is much accurate for long term periods compare to short term periods. It has long been our part of life since early of the history of human being. But it was addressed to weather forecasting only till $19^{\text {th }}$ century ${ }^{1}$. But in $20^{\text {th }}$ it gets a new dimension in the business sectors. Since 1950's a lot of research works have been done on forecasting and is still working to develop new techniques.

Corberan et al. has publicized that Exponential Smoothing is suitable under more general conditions and variance is allowed to grow with corresponding movements in the primary level ${ }^{3}$.

H. V. Ravinder examined that exponential smoothing method is quite good for large number of data to forecast. They work on various time series generated through simulation on a spreadsheet ${ }^{4}$.

P. Y. Lim and C. V. Nayar used Single Exponential Smoothing method to forecast on solar irradiance and load demand. For this, they used two different single exponential smoothing forecasting approaches to predicate for solar irradiance and load demand ${ }^{5}$.

In our paper, we discuss Exponential Smoothing Method and smoothing constant. We solve a given problem and try to find out an optimal smoothing constant by using Exponential Smoothing Method. We also calculate forecast value by using optimal smoothing constant.

We measure the accuracy by using mean absolute deviation (MAD) and mean absolute percentage error (MAPE). We observe from the empirical results of the analysis that if the data has no trend, Exponential Smoothing Forecasting Method processes as the most appropriate forecasting method for the factory because the data experiences no linear trend in it.

The rest of the paper is organized as follows. In Section II, we present an existing forecasting method which is Exponential forecasting technique. In section III, we discuss forecasting techniques by using a numerical example and also calculate forecast value by using optimal smoothing constant. Finally, we draw a conclusion.

\section{Preliminaries}

In this section, we discuss about Exponential Smoothing method .

\section{Exponential Smoothing Method}

Exponential smoothing was first introduced by Robert Goodell Brown in 1956.Extension of this method has been done by Charles C. Holt in 1957. The formula used for Exponential smoothing method is known as "Brown's simple exponential smoothing"6.

The simplest form of exponential smoothing is given by the following formula

$$
F_{t+1}=\alpha A_{t}+(1-\alpha) F_{t}
$$

Here $F_{t+1}=$ Forecast for period $t+1$,

$F_{t}=$ Forecast for the previous period (i.e.period $t$ ),

$\alpha=$ Smoothing constant (percentage) and $0<\alpha<1$,

$A_{t}=$ Actual demand or sales for the previous period

We can find the new estimate of forecasting value by using the previous equation. By using an appropriate value of $\alpha$

\footnotetext{
*Author for correspondence. e-mail: nanditabarmandu12@gmail.com
} 
we get more accurate result. This method requires an initial forecast $F_{0}$ that is assumed.

\section{Real Life Problem Discussion}

A newly launched biscuit factory produces different types of biscuits. One of them is nut-orange twisted biscuits. They supply this product all over the Bangladesh. Historical sales of nut-orange twisted biscuits data are given for some previous weeks. As it is a newly launched biscuit factory, it did not use any scientific method to find future demand of their products to produce for the purpose of sales. That's why they face some problems regarding production. If they knew what amount of newly launched biscuit has would be produced then it would be helpful for them to continue an error free production as well as they could have a good inventory management. Actual demand values (thousands) of sales are also given in Table 1 . The factory needs a reliable forecasting for demand of a finished product for the next week.

Table 1. Actual demand values (thousands) of sales

\begin{tabular}{|c|c|}
\hline Weeks & Sales \\
\hline 1 & 110 \\
\hline 2 & 115 \\
\hline 3 & 125 \\
\hline 4 & 120 \\
\hline 5 & 125 \\
\hline 6 & 120 \\
\hline 7 & 130 \\
\hline 8 & 115 \\
\hline 9 & 110 \\
\hline 10 & 130 \\
\hline
\end{tabular}

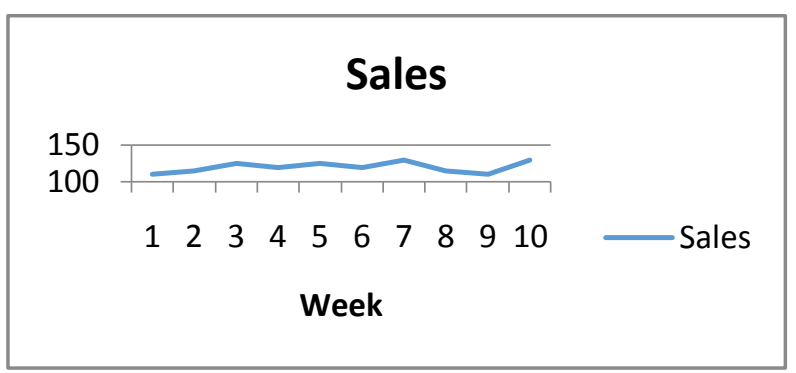

Fig. 1. Actual demand values (thousands) of sale

Fig.1. denotes that given data is a irregular data. It does not contains any trend or seasonality

\section{Forecasting by Simple Exponential smoothing method}

We use Simple Exponential smoothing method to forecast the demand value because clearly the given data indicates that data does not contain any trend as well as seasonality.

For smoothing constant $\alpha=0.1$, let the initial forecast $F_{1}=110$

Now, from Exponential smoothing method we know,

$$
\begin{gathered}
F_{2}=\alpha * A_{1}+(1-\alpha) * F_{1} \\
=0.1 * 110+(1-0.1) * 110 \\
=110
\end{gathered}
$$

Similarly, for smoothing constant $\alpha=0.1$ then we calculate forecast $\left(F_{t}\right)$ and MAD, MSE \& MAPE are also calculated for the given problem. The above process is shown in the following table:

Table 2. Solution of the given problem using Exponential Smoothing method

\begin{tabular}{|c|c|c|c|c|c|}
\hline Weeks $(t)$ & Sales $\left(A_{t}\right)$ & $F_{t}$ & $\left|A_{t}-F_{t}\right|$ & $\left(A_{t}-F_{t}\right)^{2}$ & $\frac{\left|A_{t}-F_{t}\right|}{A_{t}} * 100$ \\
\hline 1 & 110 & 110 & 0 & 0 & 0 \\
\hline 2 & 115 & 110 & 5 & 25 & 4.3472 \\
\hline 3 & 125 & 110.5 & 14.5 & 210.25 & 6.7083 \\
\hline 4 & 120 & 111.95 & 8.05 & 64.8025 & 9.796 \\
\hline 5 & 125 & 112.755 & 12.245 & 149.94 & 5.0171 \\
\hline 6 & 120 & 113.9795 & 6.0205 & 36.2464 & 11.8603 \\
\hline 7 & 130 & 114.5816 & 15.4184 & 237.7286 & 0.9768 \\
\hline 8 & 115 & 116.1234 & 1.1234 & 1.2620 & 5.4646 \\
\hline 10 & 110 & 116.0111 & 6.0111 & 36.1328 & 11.2232 \\
\hline
\end{tabular}

Table 2 shows the way how to calculate Exponential Smoothing method for the given problem. Using this table, we can compute MAD, MSE \& MAPE

We know,
Mean Absolute Deviation (MAD) is

$$
=\frac{\sum\left|A_{t}-F_{t}\right|}{n}=8.29585
$$


Mean Absolute Percentage Error (MAPE) is

$$
=\frac{\sum \frac{\left|A_{t}-F_{t}\right|}{A_{t}} * 100}{n}=6.69942
$$

Now, we take different values of smoothing constant $(\alpha)$ and for each value of $\alpha$, we calculate MAD, MAPE . After that, we find out whether MAD, MAPE give minimum value.

The above process is shown in the following Table:

Table 3. MAD, MAPE for different values of $\alpha$

\begin{tabular}{|c|c|c|}
\hline Smoothing constant $(\alpha)$ & $\begin{array}{l}\text { Mean Absolute } \\
\text { Deviation } \\
\text { (MAD) }\end{array}$ & $\begin{array}{l}\text { Mean } \\
\text { Absolute } \\
\text { Per. Error } \\
\text { (MAPE) }\end{array}$ \\
\hline 0.1 & 8.2958 & 6.6994 \\
\hline 0.2 & 7.7419 & 6.3161 \\
\hline 0.3 & 7.2998 & 5.9867 \\
\hline 0.35 & 7.1589 & 5.8793 \\
\hline 0.37 & 7.1551 & 5.8788 \\
\hline 0.38 & 7.1529 & 5.8781 \\
\hline 0.39 & 7.1505 & 5.8771 \\
\hline 0.4 & 7.1479 & 5.8758 \\
\hline 0.5 & 7.1152 & 5.8507 \\
\hline 0.57 & 7.0891 & 5.8258 \\
\hline 0.58 & 7.0853 & 5.8220 \\
\hline 0.59 & 7.0936 & 5.8282 \\
\hline 0.6 & 7.1181 & 5.8478 \\
\hline 0.7 & 7.3549 & 6.0357 \\
\hline 0.8 & 7.58 & 6.2116 \\
\hline 0.9 & 7.795 & 6.3777 \\
\hline 0.99 & 7.9799 & 6.5194 \\
\hline
\end{tabular}

From Table 3, we see that, values of MAD, MAPE are differed with the different values of $\alpha$. The variation of MAD with $\alpha$ is shown in Figure :

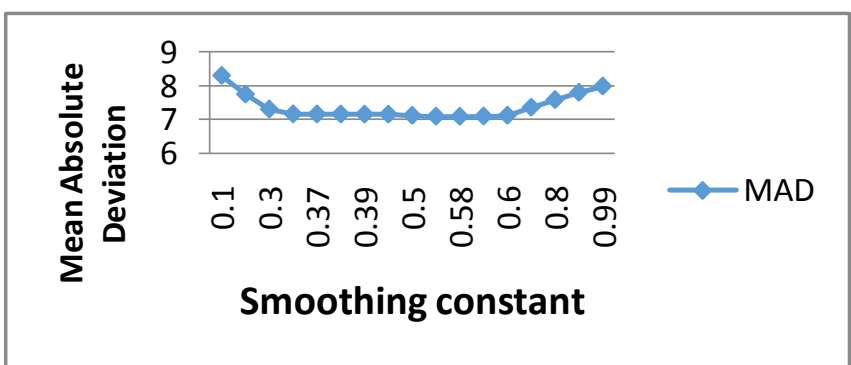

Fig. 2. Variation of Mean Absolute Deviation for different values of $\alpha$.

From Figure 2, we see that, Mean Absolute Deviation gives the minimum value for the smoothing constant $\alpha=$
0.58.The variation of Mean Absolute Percentage Error (MAPE) for different values of $\alpha$ is shown in the following figure:

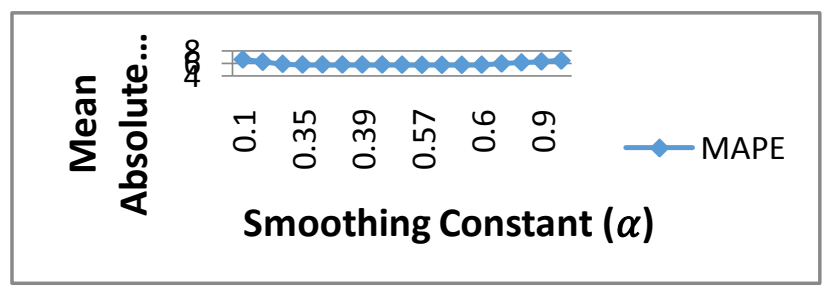

Fig. 3. Variation of MAPE for different values of $\alpha$

From Fig. 3, we see that, Mean Absolute Percentage Error gives the minimum value for the smoothing constant $\alpha=0.58$. MAD, MAPE are used for generating the optimal of $\alpha$. After analyzing Table 3 and Fig 2, Fig 3 ; we see that, the value of smoothing constants are 0.58, 0.58 for minimum MAD, MAPE respectively. Minimum values of MAD, MAPE and corresponding value of smoothing constant is given in the following Table :

Table 4. Optimal value of smoothing constant $(\alpha)$

\begin{tabular}{|c|c|c|}
\hline Criteria & Minimum value & value of $\alpha$ \\
\hline Mean Absolute & 7.0854 & 0.58 \\
Deviation(MAD) & & 0.58 \\
\hline $\begin{array}{c}\text { Mean Absolute } \\
\text { Percentage Error } \\
\text { (MAPE) }\end{array}$ & 5.8220 & \\
\hline
\end{tabular}

From the Table 4, we see that, MAD \& MAPE both give the minimum value for the value of smoothing constant $(\alpha=$ 0.58). So, 0.58 is the optimum value of smoothing constant.

Now, we calculate forecast values for the optimal smoothing constant $\alpha=0.58$

Table 5. Forecast values for optimal smoothing constant $\alpha=0.58$

\begin{tabular}{|c|c|c|}
\hline Weeks & Sales & Forecast values \\
\hline 1 & 110 & 110 \\
\hline 2 & 115 & 110 \\
\hline 3 & 125 & 112.9 \\
\hline 4 & 120 & 119.918 \\
\hline 5 & 125 & 119.9656 \\
\hline 6 & 120 & 122.8855 \\
\hline 7 & 130 & 121.2119 \\
\hline 8 & 115 & 126.309 \\
\hline 9 & 110 & 119.7498 \\
\hline 10 & 130 & 114.0949 \\
\hline 11 & - & 123.3199 \\
\hline
\end{tabular}

From Table 5, we get the forecast value for the $11^{\text {th }}$ week. Using Exponential Smoothing method the forecast value for the optimal smoothing constant $\alpha=0.58$ at the $11^{\text {th }}$ week is 123.3199.The following figure represents the comparison 
between actual sales and corresponding forecast values for optimal smoothing constant.

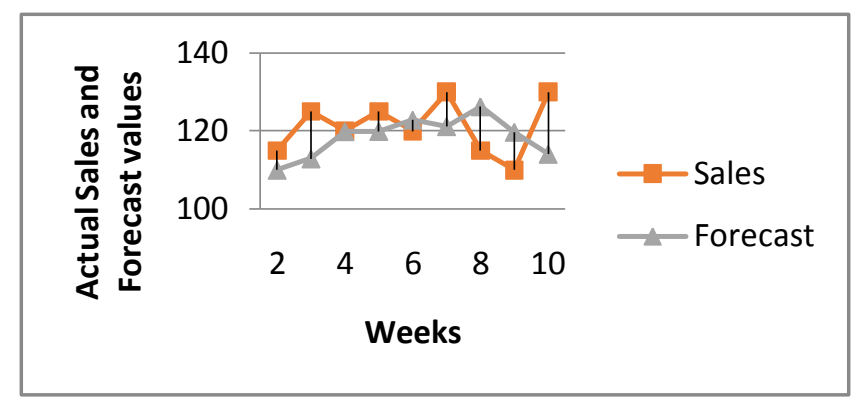

Fig. 4. Comparison of actual sales and forecast value

Fig.4 represents the comparison between actual sales and forecast value for optimal smoothing constant.

Exponential Model has the highest degree of model adequacy, and forecast accuracy. In terms of interpretability, we concluded that for the given data we analyzed Exponential Smoothing Method may be a strongly suitable model, because sales seems to maintain no linear trend. If linear trend exist Holt's method will be more suitable than Exponential Smoothing Method.

\section{Conclusion}

There are many existing techniques for forecasting. The pictorial description says that our given data does not contain any trend and also does not contain any seasonality. If data contains any trend we will use Holt's method and if it contains any seasonality than we will use Winter's forecasting method. Though data does not have any trend and seasonality, so we use Simple Exponential Smoothing method to forecast the sales value of $11^{\text {th }}$ week by using optimal smoothing constant.

\section{References}

1. Armstrong J. S., 2007. Significance tests harm progress in forecasting, International Journal of Forecasting, 23(2), 321 327.

2. Bermudez J.D, J. V Segura., and E.Velcher, 2006. Improving Demand Forecasting Accuracy Using Nonlinear Programming Software,Journal of the Operational Research Society, 57, 94-100.
3. Corberan-Vallet A., J. D Bermudez., and E. Vercher, 2011. Forecasting correlated time series with exponential smoothing models, International Journal of Forecasting, 27, 252-265.

4. Ravinder, H. V., 2013. Determining the Optimal Values of Exponential Smoothing Constants - Does Solver Really Work, American Journal of Business Education, 6(3), May/June.

5. Lim, P. Y., and C. V. Nayar, 2012. Solar Irradiance and Load Demand Forecasting based onSingle Exponential Smoothing Method, International Journal of Engineering and Technology, 4(4).

6. Gardner, E.S., 2006. Exponential Smoothing: The State of the Art, Part-II, International Journal of Forecasting, 22, 637-666.

7. Mikhail Aseev., Nemeshaev., Sergei and Nesterov Alexander, 2016. Forecasting Cash Withdrawals in the ATM Network Using a Combined Model based on the Holt-Winters Method and Markov Chains, International Journal of Applied Engineering Research 11(11), 7577-7582 (C) Research India Publications, http://www.ripublication.com.

8. Harvey, A. C. 1988,A Unified View of Statistical Forecasting Procedures, Journal of Forecasting, 3, 245-275.

9. Hyndman, R. J., A. B Koehler., R. D Snyder, and S. Grose, 2002, A state space framework for automatic forecasting using exponential smoothing methods, International Journal of Forecasting, 18, 439-454.

10. McKenzi, E., and E. S. Gardner, 2010, Damped trend exponential smoothing: A modeling viewpoint, International Journal of Forecasting, 26, 661-665.

11. Taylor, J. W., 2004. Smooth Transition Exponential smoothing, International Journal of Forecasting, 23,385-394.

12. Taylor James W. 2004, Volatility Forecasting with Smooth Transition Exponential Smoothing, International Journal of Forecasting, 20, 273-286.

13. Singh* Amrit Pal, Gaur Manoj Kumar, Kasdekar Dinesh Kumar and Agrawal Sharad,2015,A Study of Time Series Model for Forecasting of Boot in Shoe Industry , International Journal of Hybrid Information Technology, 8(8), 143-152. 